Supplement of:

\title{
In situ Ozone Production is highly sensitive to Volatile Organic Compounds in the Indian Megacity of Delhi
}

\section{Beth S. Nelson et al.}

5 Correspondence to: Beth S. Nelson (bsn502@york.ac.uk)
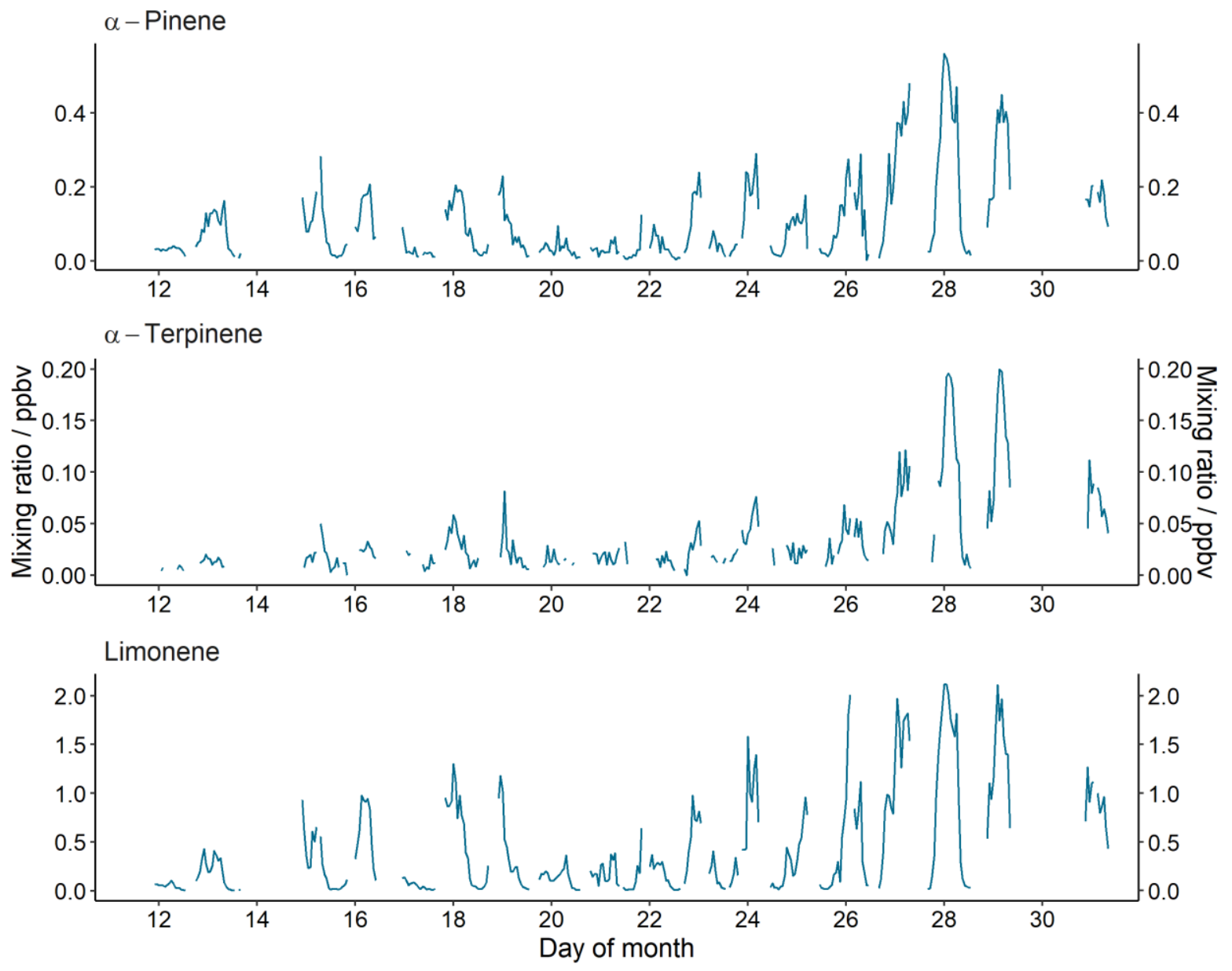

Figure S1: Selected timeseries for monoterpenes measured during October, 2018 at Indira Gandhi Delhi Technical University for Women. Top to bottom: $\alpha$-pinene, $\alpha$-terpinene, limonene. 

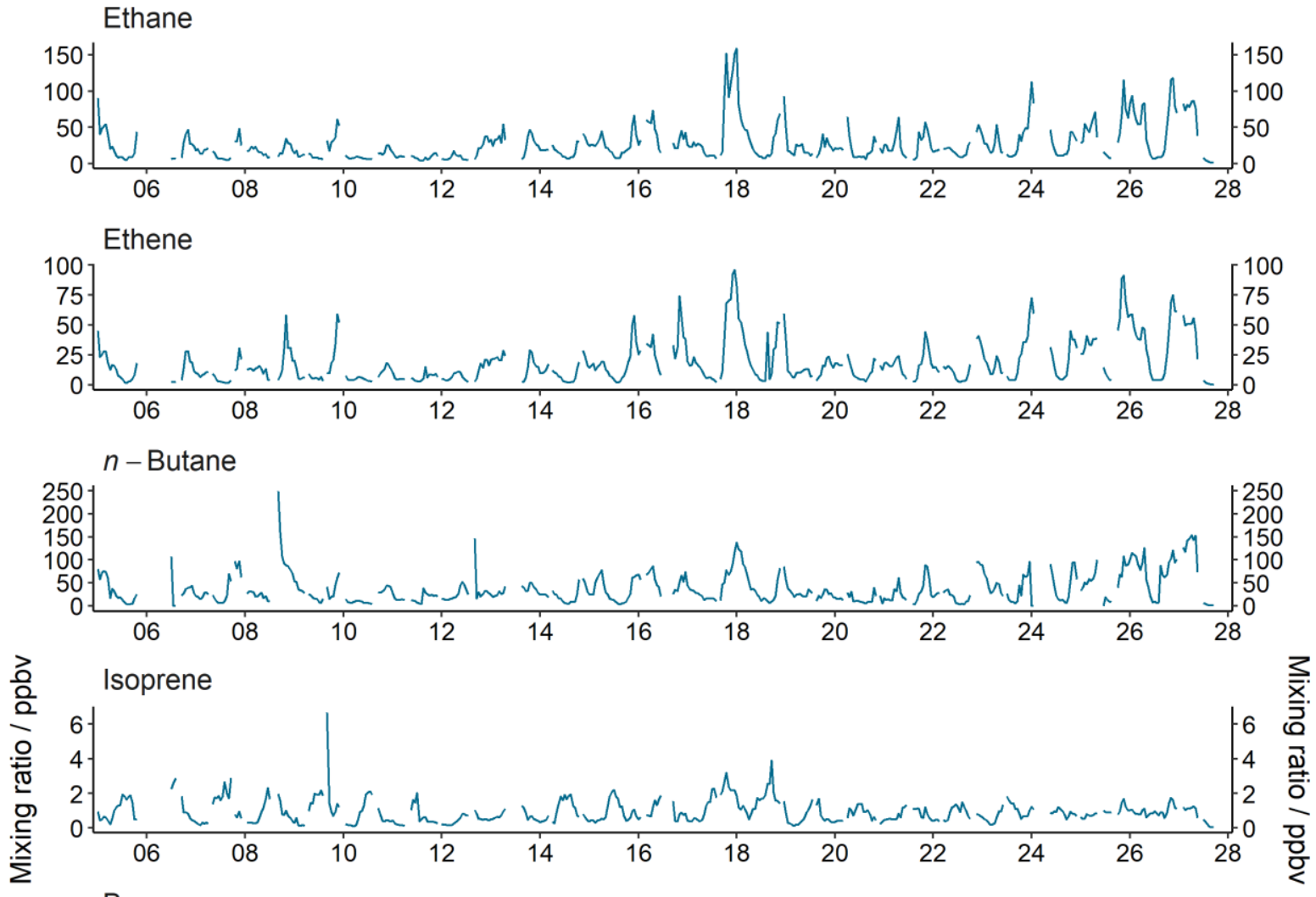

Benzene

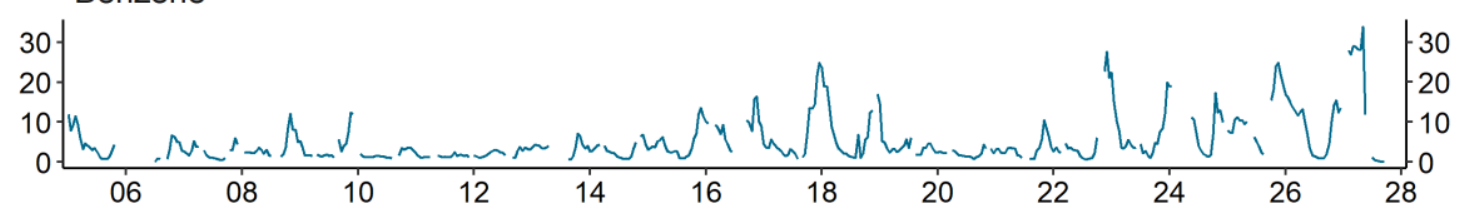

Ethanol
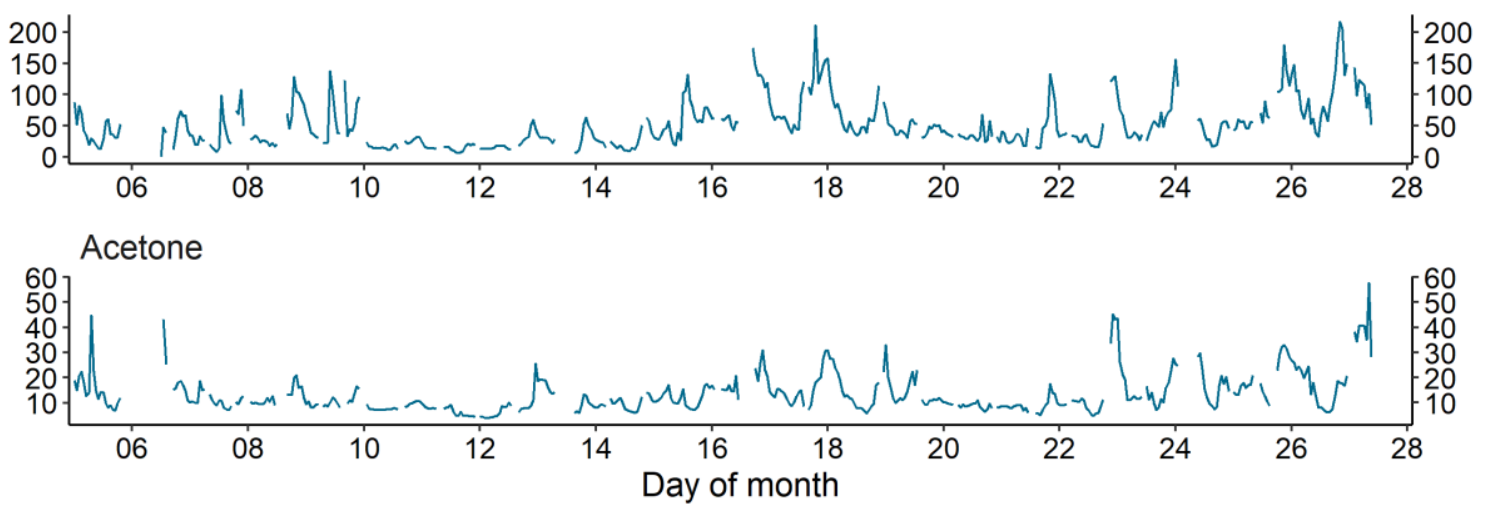

10 Figure S2: Selected timeseries for VOCs measured during October, 2018 at Indira Gandhi Delhi Technical University for Women. Top to bottom: ethane, ethene, $n$-butane, isoprene, benzene, ethanol, acetone. 
Table S1: List of VOCs included in model, along with campaign averaged mixing ratios and proxy mechanisms for species not found in the master chemical mechanism (MCM). Rate constants for additional species to those in the MCM can be found on IUPAC (IUPAC, accessed in 2019) or in Atkinson and Arey, 2003.

\begin{tabular}{|c|c|c|c|c|c|c|}
\hline Species & Class & $\begin{array}{l}\text { Campa } \\
\text { Mixing } \\
\text { Mean }\end{array}$ & $\begin{array}{l}\text { gn } \\
\text { Ratios } \\
\text { Min. }\end{array}$ & $\begin{array}{l}\text { verage } \\
\text { opbv } \\
\text { Max. }\end{array}$ & $\begin{array}{l}\text { Proxy Mechanism used } \\
\text { for species not in the } \\
\text { MCM }\end{array}$ & Instrument \\
\hline $\begin{array}{l}\text { Ethanol } \\
\text { Methanol }\end{array}$ & Alcohol & $\begin{array}{l}50.98 \\
49.87\end{array}$ & $\begin{array}{l}29.69 \\
21.1\end{array}$ & $\begin{array}{l}92 \\
81.71\end{array}$ & $\begin{array}{l}- \\
-\end{array}$ & $\begin{array}{l}\text { (DC)-GC-FID } \\
\text { (DC)-GC-FID }\end{array}$ \\
\hline Ethane & Alkane & 27.09 & 7.09 & 51.63 & - & (DC)-GC-FID \\
\hline Isobutane & & 19.89 & 5.96 & 34.95 & - & (DC)-GC-FID \\
\hline Isopentane & & 15.86 & 3.97 & 36 & - & (DC)-GC-FID \\
\hline$n$-Butane & & 37.21 & 6.7 & 69.21 & - & (DC)-GC-FID \\
\hline$n$-Decane & & 1.07 & 0.2 & 1.99 & - & $\mathrm{GC} \times \mathrm{GC}-\mathrm{FID}$ \\
\hline$n$-Dodecane & & 1.09 & 0.16 & 1.87 & - & $\mathrm{GC} \times \mathrm{GC}-\mathrm{FID}$ \\
\hline$n$-Heptane & & 1.18 & 0.2 & 2.93 & - & (DC)-GC-FID \\
\hline$n$-Hexane & & 1.63 & 0.3 & 3.69 & - & (DC)-GC-FID \\
\hline$n$-Nonane & & 1.35 & 0.24 & 2.67 & - & $\mathrm{GC} \times \mathrm{GC}-\mathrm{FID}$ \\
\hline$n$-Octane & & 0.54 & 0.09 & 1.33 & - & (DC)-GC-FID \\
\hline$n$-Pentane & & 6.05 & 1.4 & 13.48 & - & (DC)-GC-FID \\
\hline$n$-Tetradecane & & 1.42 & 0.41 & 2.28 & $n$-Dodecane & $\mathrm{GC} \times \mathrm{GC}-\mathrm{FID}$ \\
\hline$n$-Tridecane & & 2.93 & 0.52 & 4.67 & $n$-Dodecane & GC $\times$ GC-FID \\
\hline$n$-Undecane & & 0.54 & 0.09 & 0.95 & - & $\mathrm{GC} \times \mathrm{GC}-\mathrm{FID}$ \\
\hline Propane & & 39.12 & 9.84 & 68.95 & - & (DC)-GC-FID \\
\hline 1,2-Butadiene & Alkene & 0.28 & 0.07 & 0.45 & 1,3-Butadiene & (DC)-GC-FID \\
\hline 1,3-Butadiene & & 0.7 & 0.07 & 1.65 & - & (DC)-GC-FID \\
\hline But-1-ene & & 1.47 & 0.21 & 3.02 & - & (DC)-GC-FID \\
\hline cis-But-2-ene & & 1.03 & 0.2 & 2.19 & - & (DC)-GC-FID \\
\hline Ethene & & 18.34 & 3.14 & 39.82 & - & (DC)-GC-FID \\
\hline Isopropene & & 2.22 & 0.32 & 4.65 & - & (DC)-GC-FID \\
\hline Pent-1-ene & & 0.34 & 0.06 & 0.77 & - & (DC)-GC-FID \\
\hline Propene & & 5.26 & 0.64 & 11.3 & - & (DC)-GC-FID \\
\hline trans-But-2-ene & & 1.07 & 0.16 & 2.36 & - & (DC)-GC-FID \\
\hline trans-Pent-2-ene & & 0.53 & 0.09 & 1.4 & - & (DC)-GC-FID \\
\hline
\end{tabular}




\begin{tabular}{|c|c|c|c|c|c|c|}
\hline $\begin{array}{l}\text { Acetylene } \\
\text { Propyne }\end{array}$ & Alkyne & $\begin{array}{l}8.91 \\
0.38\end{array}$ & $\begin{array}{l}2.16 \\
0.09\end{array}$ & $\begin{array}{l}20.2 \\
0.82\end{array}$ & $\begin{array}{l}- \\
\text { Acetylene }\end{array}$ & $\begin{array}{l}\text { (DC)-GC-FID } \\
\text { (DC)-GC-FID }\end{array}$ \\
\hline 1,2,3,4-Tetramethylbenzene & Aromatic & 0.21 & 0.01 & 0.5 & 1,2,3-Trimethylbenzene & GC $\times$ GC-FID \\
\hline 1,2,3,5-Tetramethylbenzene & & 0.28 & 0.02 & 0.64 & 1,2,3-Trimethylbenzene & $\mathrm{GC} \times \mathrm{GC}-\mathrm{FID}$ \\
\hline 1,2,3-Trimethylbenzene & & 0.64 & 0.06 & 1.48 & - & $\mathrm{GC} \times \mathrm{GC}-\mathrm{FID}$ \\
\hline 1,2,4,5-Tetramethylbenzene & & 0.16 & 0.02 & 0.32 & 1,2,4-Trimethylbenzene & $\mathrm{GC} \times \mathrm{GC}-\mathrm{FID}$ \\
\hline 1,2,4-Trimethylbenzene & & 1.86 & 0.2 & 4.3 & - & $\mathrm{GC} \times \mathrm{GC}-\mathrm{FID}$ \\
\hline 1,2-Diethylbenzene & & 0.18 & 0.02 & 0.39 & $o$-Ethyltoluene & $\mathrm{GC} \times \mathrm{GC}-\mathrm{FID}$ \\
\hline 1,2-Dimethyl-4-ethylbenzene & & 0.35 & 0.02 & 0.84 & $\begin{array}{l}\text { 3,5-Dimethyl-1- } \\
\text { ethylbenzene }\end{array}$ & $\mathrm{GC} \times \mathrm{GC}-\mathrm{FID}$ \\
\hline 1,3,5-Trimethylbenzene & & 0.61 & 0.05 & 1.41 & - & $\mathrm{GC} \times \mathrm{GC}-\mathrm{FID}$ \\
\hline 1,3-Diethyl-2-ethylbenzene & & 0.1 & 0.02 & 0.21 & $\begin{array}{l}\text { 3,5-Dimethyl-1- } \\
\text { ethylbenzene }\end{array}$ & $\mathrm{GC} \times \mathrm{GC}-\mathrm{FID}$ \\
\hline 1,3-Diethylbenzene & & 0.2 & 0.02 & 0.44 & $m$-Ethyltoluene & $\mathrm{GC} \times \mathrm{GC}-\mathrm{FID}$ \\
\hline 1,4-Diethylbenzene & & 0.51 & 0.04 & 1.20 & $p$-Ethyltoluene & $\mathrm{GC} \times \mathrm{GC}-\mathrm{FID}$ \\
\hline 1-Methyl-3-propylbenzene & & 0.10 & 0.01 & 0.21 & Propylbenzene & $\mathrm{GC} \times \mathrm{GC}-\mathrm{FID}$ \\
\hline 1-Methyl-4-propylbenzene & & 0.14 & 0.01 & 0.33 & Propylbenzene & $\mathrm{GC} \times \mathrm{GC}-\mathrm{FID}$ \\
\hline 1-Methylpropylbenzene & & 0.17 & 0.02 & 0.36 & Propylbenzene & $\mathrm{GC} \times \mathrm{GC}-\mathrm{FID}$ \\
\hline 2,3-Dimethyl-1-ethylbenzene & & 0.09 & 0.01 & 0.22 & $\begin{array}{l}\text { 3,5-Dimethyl-1- } \\
\text { ethylbenzene }\end{array}$ & $\mathrm{GC} \times \mathrm{GC}-\mathrm{FID}$ \\
\hline 2,4-Dimethyl-1-ethylbenzene & & 0.17 & 0.02 & 0.4 & $\begin{array}{l}\text { 3,5-Dimethyl-1- } \\
\text { ethylbenzene }\end{array}$ & $\mathrm{GC} \times \mathrm{GC}-\mathrm{FID}$ \\
\hline 2-Methylpropylbenzene & & 0.16 & 0.02 & 0.36 & Propylbenzene & $\mathrm{GC} \times \mathrm{GC}-\mathrm{FID}$ \\
\hline Benzene & & 5.26 & 1.15 & 10.05 & - & (DC)-GC-FID \\
\hline Ethylbenzene & & 2.09 & 0.36 & 4.88 & - & (DC)-GC-FID \\
\hline Isoproylbenzene & & 0.29 & 0.03 & 0.72 & - & $\mathrm{GC} \times \mathrm{GC}-\mathrm{FID}$ \\
\hline$m$-Xylene & & 2.19 & 0.32 & 6.02 & - & (DC)-GC-FID \\
\hline$n$-Butylbenzene & & 0.51 & 0.04 & 1.2 & Propylbenzene & $\mathrm{GC} \times \mathrm{GC}-\mathrm{FID}$ \\
\hline$o$-Ethyltoluene & & 0.65 & 0.08 & 1.47 & - & $\mathrm{GC} \times \mathrm{GC}-\mathrm{FID}$ \\
\hline$o$-Xylene & & 1.85 & 0.3 & 4.86 & - & (DC)-GC-FID \\
\hline$p$-Ethyltoluene & & 1.78 & 0.27 & 4.13 & - & $\mathrm{GC} \times \mathrm{GC}-\mathrm{FID}$ \\
\hline Propylbenzene & & 0.39 & 0.06 & 0.87 & - & $\mathrm{GC} \times \mathrm{GC}-\mathrm{FID}$ \\
\hline$p$-Xylene & & 2.19 & 0.32 & 6.02 & - & (DC)-GC-FID \\
\hline
\end{tabular}




\begin{tabular}{|c|c|c|c|c|c|c|}
\hline $\begin{array}{l}\text { Styrene } \\
\text { Toluene }\end{array}$ & & $\begin{array}{l}0.9 \\
14.09\end{array}$ & $\begin{array}{l}0.06 \\
2.62\end{array}$ & $\begin{array}{l}1.69 \\
30.09\end{array}$ & - & $\begin{array}{l}\text { GC } \times \text { GC-FID } \\
(D C)-G C-F I D\end{array}$ \\
\hline Acetaldehyde & Carbonyl & 8.3 & 2.93 & 11.89 & - & PTR-QiTOF-MS \\
\hline Acetone & & 13.24 & 7.44 & 18.64 & - & (DC)-GC-FID \\
\hline Cyclohexane & & 0.35 & 0.12 & 0.55 & - & PTR-QiTOF-MS \\
\hline Ethylacetate & & 8.48 & 2.04 & 14.14 & - & $\mathrm{GC} \times \mathrm{GC}-\mathrm{FID}$ \\
\hline Formaldehyde & & 14.29 & 8.69 & 18.25 & - & PTR-QiTOF-MS \\
\hline Hexan-2-one & & 0.48 & 0.15 & 0.67 & - & PTR-QiTOF-MS \\
\hline Hexan-3-one & & 0.48 & 0.15 & 0.67 & - & PTR-QiTOF-MS \\
\hline Methacrolein & & 1.01 & 0.49 & 1.46 & - & PTR-QiTOF-MS \\
\hline Methyl isobutyl ketone & & 0.48 & 0.15 & 0.67 & - & PTR-QiTOF-MS \\
\hline Methyl propyl ketone & & 1.64 & 0.73 & 2.28 & - & PTR-QiTOF-MS \\
\hline Methyl vinyl ketone & & 3.59 & 1.2 & 5.29 & - & PTR-QiTOF-MS \\
\hline Methyl vinyl ketone & & 1.01 & 0.49 & 1.46 & - & PTR-QiTOF-MS \\
\hline Isoprene & Isoprene & 0.94 & 0.44 & 1.57 & - & (DC)-GC-FID \\
\hline$\alpha$-Phellandrene & Monoterpene & 0.09 & 0.02 & 0.15 & $\alpha$-Pinene & $\mathrm{GC} \times \mathrm{GC}-\mathrm{FID}$ \\
\hline 3-Carene & & 0.08 & 0.02 & 0.15 & $\alpha$-Pinene & $\mathrm{GC} \times \mathrm{GC}-\mathrm{FID}$ \\
\hline Camphene & & 0.09 & 0.01 & 0.16 & $\beta$-Pinene & $\mathrm{GC} \times \mathrm{GC}-\mathrm{FID}$ \\
\hline Limonene & & 0.38 & 0.02 & 0.79 & - & $\mathrm{GC} \times \mathrm{GC}-\mathrm{FID}$ \\
\hline$m$-Cymene & & 0.53 & 0.04 & 1.03 & $m$-Ethyltoluene & GC $\times$ GC-FID \\
\hline Myrcene & & 0.08 & 0.01 & 0.14 & Limonene & $\mathrm{GC} \times \mathrm{GC}-\mathrm{FID}$ \\
\hline$o$-Cymene & & 0.06 & 0.01 & 0.11 & $o$-Ethyltoluene & $\mathrm{GC} \times \mathrm{GC}-\mathrm{FID}$ \\
\hline$p$-Cymene & & 0.53 & 0.04 & 1.03 & $p$-Ethyltoluene & $\mathrm{GC} \times \mathrm{GC}-\mathrm{FID}$ \\
\hline Sabinene & & 0.06 & 0.02 & 0.13 & $\beta$-Pinene & $\mathrm{GC} \times \mathrm{GC}-\mathrm{FID}$ \\
\hline Terpinolene & & 0.07 & 0 & 0.15 & $\beta$-Pinene & $\mathrm{GC} \times \mathrm{GC}-\mathrm{FID}$ \\
\hline$\alpha$-Pinene & & 0.09 & 0.01 & 0.18 & - & $\mathrm{GC} \times \mathrm{GC}-\mathrm{FID}$ \\
\hline$\alpha$-Terpinene & & 0.03 & 0.01 & 0.06 & $\alpha$-Pinene & $\mathrm{GC} \times \mathrm{GC}-\mathrm{FID}$ \\
\hline$\beta$-Ocimene & & 0.31 & 0.02 & 0.67 & Limonene & $\mathrm{GC} \times \mathrm{GC}-\mathrm{FID}$ \\
\hline$\beta$-Pinene & & 0.05 & 0.01 & 0.09 & - & $\mathrm{GC} \times \mathrm{GC}-\mathrm{FID}$ \\
\hline$\gamma$-terpinene & & 0.05 & 0.01 & 0.1 & $\alpha$-Pinene & $\mathrm{GC} \times \mathrm{GC}-\mathrm{FID}$ \\
\hline
\end{tabular}




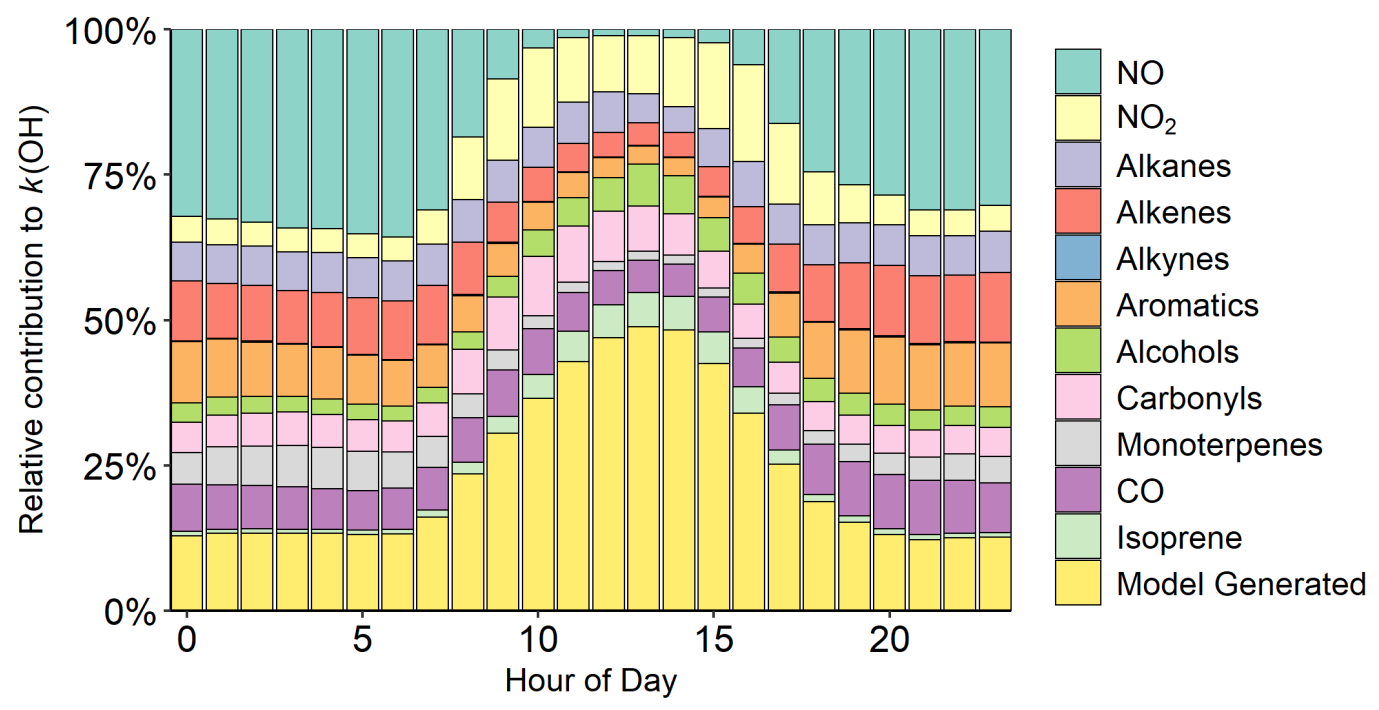

Figure S3: Relative contributions of $\mathrm{NO}, \mathrm{NO}_{2}, \mathrm{CO}$, VOC classes and model generated species to $k(\mathrm{OH})$ per hour.

Table S2: $\Delta \mathrm{P}\left(\mathrm{O}_{3}\right)_{\text {increm }}$ for 77 VOCs, averaged between 08:00 and 12:00. The 10 highest values are shown in bold.

\begin{tabular}{ll}
\hline Species & $\Delta \mathrm{P}\left(\mathrm{O}_{3}\right)_{\text {increm }} / \mathrm{ppb} \mathrm{hr}^{-1}$ \\
\hline$\alpha$-Phellandrene & $\mathbf{0 . 3 0}$ \\
$\alpha$-Pinene & 0.02 \\
$\alpha$-Terpinene & $\mathbf{0 . 6 6}$ \\
1,2-Butadiene & 0.02 \\
Benzene & 0.02 \\
$\beta$-Ocimene & $\mathbf{0 . 2 6}$ \\
$\beta$-Pinene & 0.00 \\
But-1-ene & 0.12 \\
Ethyne & 0.00 \\
Ethene & $\mathbf{0 . 4 0}$ \\
Ethanol & $\mathbf{0 . 3 0}$ \\
Ethane & 0.01 \\
Propyne & 0.00 \\
Propene & $\mathbf{0 . 3 5}$ \\
Propane & 0.07 \\
1,3-Butadiene & 0.07 \\
Isoprene & $\mathbf{0 . 9 4}$
\end{tabular}




\begin{tabular}{|c|c|}
\hline 3-Carene & 0.03 \\
\hline Cis-But-2-ene & 0.19 \\
\hline Methanol & 0.08 \\
\hline Camphene & 0.01 \\
\hline $\mathrm{CO}$ & 0.28 \\
\hline 1,2-Diethylbenzene & 0.01 \\
\hline 1,3-Diethylbenzene & 0.02 \\
\hline 1,4-Diethylbenzene & 0.02 \\
\hline 1,2-Dimethyl-4-ethylbenzene & 0.06 \\
\hline 1,3-Dimethyl-2-ethylbenzene & 0.03 \\
\hline 2,3-Dimethyl-1-ethylbenezne & 0.02 \\
\hline 2,4-Dimethyl-1-ethylbenzene & 0.03 \\
\hline Ethylbenzene & 0.06 \\
\hline$\gamma$-Terpinene & 0.03 \\
\hline Hex-2-one & 0.00 \\
\hline Hex-3-one & 0.00 \\
\hline Isobutane & 0.15 \\
\hline Isopentane & 0.14 \\
\hline Isopropylbenzene & 0.01 \\
\hline Limonene & 0.09 \\
\hline$m$-Cymene & 0.03 \\
\hline 1-Methyl-3-propylbenzene & 0.00 \\
\hline 1-Methyl-4-propylbenzene & 0.00 \\
\hline 1-Methylpropylbenzene & 0.00 \\
\hline 2-Methylpropylbenzene & 0.00 \\
\hline Methylpropene & 0.23 \\
\hline$m$-Xylene & 0.21 \\
\hline Myrcene & 0.08 \\
\hline$n$-Butylbenzene & 0.02 \\
\hline$n$-Decane & 0.01 \\
\hline$n$-Undecane & 0.00 \\
\hline$n$-Dodecane & 0.01 \\
\hline$n$-Tridecane & 0.0 \\
\hline
\end{tabular}




\begin{tabular}{ll}
$n$-Tetradecane & 0.04 \\
$n$-Butane & 0.21 \\
$n$-Pentane & 0.07 \\
$n$-Hexane & 0.01 \\
$n$-Heptane & 0.01 \\
$n$-Octane & 0.00 \\
$n$-Nonane & 0.01 \\
$o$-Cymene & 0.00 \\
$o$-Ethyltoluene & 0.04 \\
$o$-Xylene & 0.14 \\
Propylbenzene & 0.01 \\
$p$-Cymene & 0.02 \\
Pent-1-ene & 0.03 \\
$p$-Ethyltoluene & 0.08 \\
$p$-Xylene & 0.13 \\
Sabinene & 0.01 \\
Styrene & 0.01 \\
trans-But-2-ene & 0.22 \\
1,2,3,4-Tetramethylbenzene & 0.04 \\
1,2,3,5-Tetramethylbenzene & 0.06 \\
1,2,4,5-Tetramethylbenzene & 0.04 \\
1,2,3-Trimethylbenzene & 0.09 \\
1,2,4-Trimethylbenzene & $\mathbf{0 . 2 9}$ \\
1,3,5-Trimethylbenzene & 0.12 \\
Toluene & $\mathbf{0 . 3 7}$ \\
trans-Pent-2-ene & 0.11 \\
Terpinolene & 0.03 \\
\hline & \\
&
\end{tabular}

Table S3: Description of source sectors using in section 3.8. IPCC 1996 codes and descriptions taken from IPCC, 1996.

\begin{tabular}{|l|l|l|l|}
\hline Source & \multicolumn{2}{|l|}{ IPCC code (1996) } & Description \\
\hline Road Transport (RT) & $1 \mathrm{~A} 3 \mathrm{~b}$ & $1 \mathrm{~A} 3 \mathrm{~b}$ & $\begin{array}{l}\text { All combustion and evaporative emissions from fuel use in road } \\
\text { vehicles, including agricultural vehicles on highways. }\end{array}$ \\
\hline
\end{tabular}




\begin{tabular}{|c|c|c|c|}
\hline & & $\mathrm{i}$ & $\begin{array}{l}\text { Cars: automobiles designed primary for transport of persons, with a } \\
\text { capacity of } 12 \text { or fewer. Gross vehicle weight rating of } 3900 \mathrm{~kg} \text { or } \\
\text { less. Includes passenger vehicles with and without 3-way catalysts. }\end{array}$ \\
\hline & & ii & $\begin{array}{l}\text { Light duty trucks: Vehicles with a gross vehicle weight of } 3900 \mathrm{~kg} \\
\text { or less, designed primarily for the transportation of light-weight } \\
\text { cargo or equipped with special features such as four-wheel drive for } \\
\text { off-road operation. Includes light duty trucks with and without 3- } \\
\text { way catalysts. }\end{array}$ \\
\hline & & iii & $\begin{array}{l}\text { Heavy Duty trucks and buses: Any vehicle rated at more than } 3900 \\
\mathrm{~kg} \text { gross vehicle weight or designed to carry more than } 12 \text { persons } \\
\text { at a time. }\end{array}$ \\
\hline & & iv & $\begin{array}{l}\text { Motorcycles: Any motor vehicle designed to travel with not more } \\
\text { than three wheels in contact with the ground, and weighing less than } \\
680 \mathrm{~kg} \text {. }\end{array}$ \\
\hline & & $\mathrm{v}$ & Evaporative emissions from vehicles. \\
\hline \multirow{2}{*}{$\begin{array}{l}\text { Railways, Pipelines and } \\
\text { Off-Road Transport } \\
\text { (RPORT) }\end{array}$} & \multirow[t]{2}{*}{$1 \mathrm{~A} 3 \mathrm{c}+1 \mathrm{~A} 3 \mathrm{e}$} & $1 \mathrm{~A} 3 \mathrm{c}$ & $\begin{array}{l}\text { Fuel combustion emissions from railways, including both freight } \\
\text { and passenger traffic routes. }\end{array}$ \\
\hline & & $1 \mathrm{~A} 3 \mathrm{e}$ & $\begin{array}{l}\text { Combustion emissions from all remaining transport activities } \\
\text { including pipeline transportation, ground activities in airports and } \\
\text { harbours, and off-road activities not reported under agriculture or } \\
\text { manufacturing industries and construction. }\end{array}$ \\
\hline \multirow[t]{3}{*}{$\begin{array}{l}\text { Energy for Buildings } \\
\text { (EB) }\end{array}$} & \multirow[t]{3}{*}{$1 \mathrm{~A} 4$} & $1 \mathrm{~A} 4 \mathrm{a}$ & $\begin{array}{l}\text { Emissions from fuel combustion in commercial and institutional } \\
\text { buildings. }\end{array}$ \\
\hline & & $1 \mathrm{~A} 4 \mathrm{~b}$ & All emissions from residential fuel combustion in households. \\
\hline & & $1 \mathrm{~A} 4 \mathrm{c}$ & $\begin{array}{l}\text { Emissions from fuel combustion in agriculture, forestry or domestic } \\
\text { inland coastal and deep-sea fishing. Includes traction vehicles, } \\
\text { pump fuel use, grain drying, horticultural greenhouse and other } \\
\text { agriculture, forestry or fishing related fuel use. }\end{array}$ \\
\hline \multirow[t]{4}{*}{$\begin{array}{l}\text { Combustion for } \\
\text { Manufacturing (CM) }\end{array}$} & \multirow[t]{4}{*}{$1 \mathrm{~A} 2$} & $1 \mathrm{~A} 2$ & $\begin{array}{l}\text { Emissions from combustion of fuels in industry including } \\
\text { combustion for the generation of electricity and heat. }\end{array}$ \\
\hline & & $1 \mathrm{~A} 2 \mathrm{a}$ & Iron and steel. \\
\hline & & $1 \mathrm{~A} 2 \mathrm{~b}$ & Non-ferrous metals. \\
\hline & & $1 \mathrm{~A} 2 \mathrm{c}$ & Chemicals. \\
\hline
\end{tabular}




\begin{tabular}{|c|c|c|c|}
\hline & & $1 \mathrm{~A} 2 \mathrm{~d}$ & Pulp, paper and print. \\
\hline & & $1 \mathrm{~A} 2 \mathrm{e}$ & Food processing, beverages and tobacco. \\
\hline & & $1 \mathrm{~A} 2 \mathrm{f}$ & $\begin{array}{l}\text { Other emissions from fuel combustion in industry, including from } \\
\text { the construction branch. }\end{array}$ \\
\hline Process emissions (PE) & 2 & $2 \mathrm{~A}$ & Emissions from industrial processes: mineral products. \\
\hline & & $2 \mathrm{~A} 1$ & Cement production. \\
\hline & & $2 \mathrm{~A} 2$ & Lime production. \\
\hline & & $2 \mathrm{~A} 3$ & Limestone and dolomite use. \\
\hline & & $2 \mathrm{~A} 4$ & Soda ash production and use. \\
\hline & & $2 \mathrm{~A} 5$ & Asphalt roofing. \\
\hline & & $2 \mathrm{~A} 6$ & Road paving with asphalt. \\
\hline & & $2 \mathrm{~A} 7$ & Other. \\
\hline & & $2 \mathrm{~B}$ & Emissions from chemical industry. \\
\hline & & 2B1 & Ammonia production. \\
\hline & & 2B2 & Nitric acid production. \\
\hline & & 2B3 & Adipic acid production. \\
\hline & & 2B4 & Carbide production. \\
\hline & & 2B5 & Other. \\
\hline & & $2 \mathrm{C}$ & Emissions from metal production industry. \\
\hline & & $2 \mathrm{C} 1$ & Iron and steel production. \\
\hline & & $2 \mathrm{C} 2$ & Ferroalloys production. \\
\hline & & $2 \mathrm{C} 3$ & Aluminium production. \\
\hline & & $2 \mathrm{C} 4$ & $\mathrm{SF}_{6}$ used in aluminium and magnesium foundries. \\
\hline & & $2 \mathrm{C} 5$ & Other. \\
\hline & & $2 \mathrm{D}$ & Emissions from other production industries. \\
\hline & & 2D1 & Pulp and paper. \\
\hline & & $2 \mathrm{D} 2$ & Food and drink. \\
\hline & & $2 \mathrm{E}$ & Production of halocarbons and sulfur hexafluoride. \\
\hline & & 2E1 & By-product emissions. \\
\hline & & $2 \mathrm{E} 2$ & Fugitive emissions. \\
\hline & & 2E3 & Other. \\
\hline & & $2 \mathrm{~F}$ & Consumption of halocarbons and sulfur hexafluoride. \\
\hline
\end{tabular}




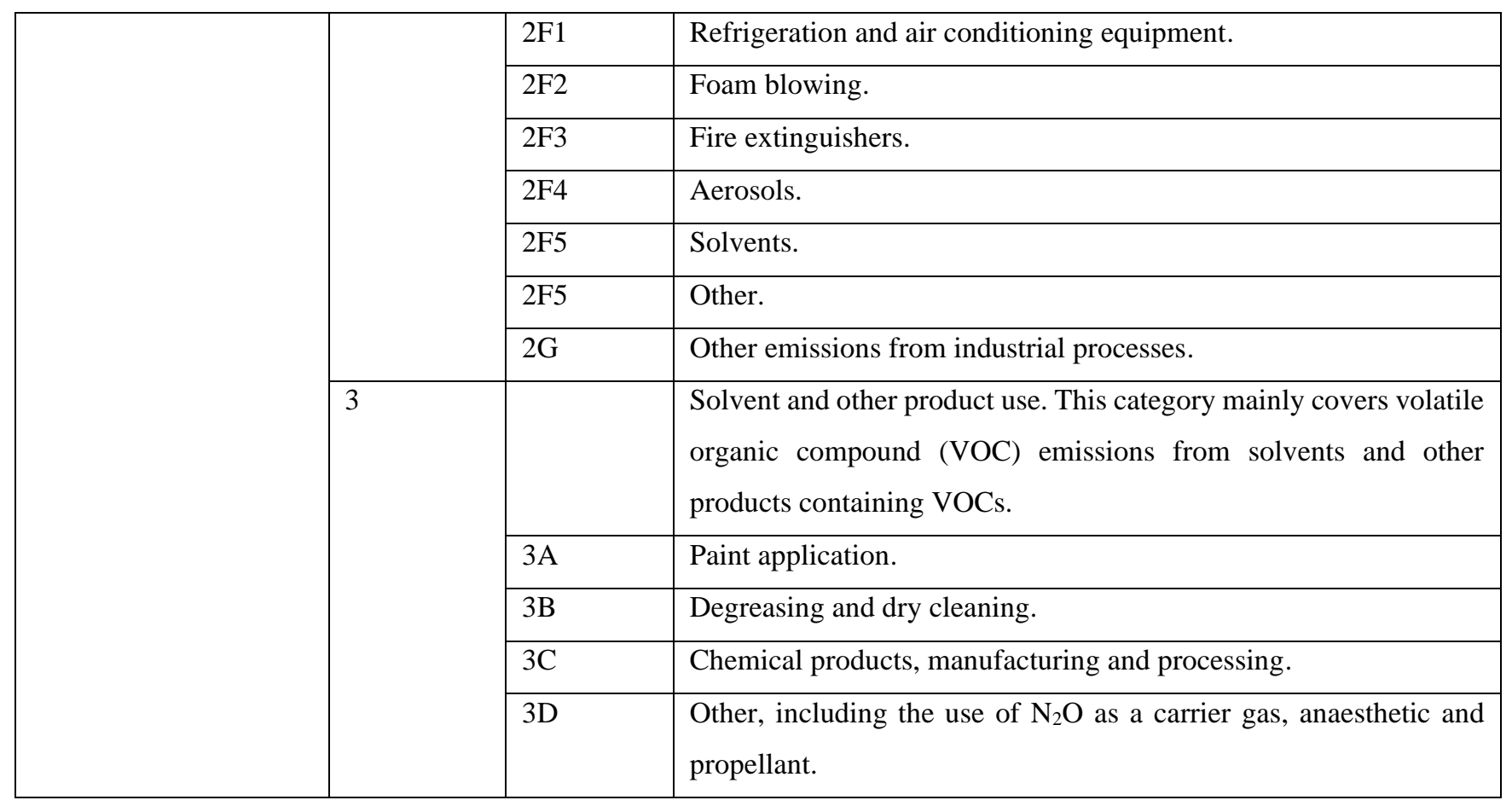

\section{References}

Atkinson, R. and Arey, J.: Atmospheric Degradation of Volatile Organic Compounds Atmospheric Degradation of Volatile Organic Compounds, Chem. Rev., 103(3), 4605-4638, doi:10.1021/cr0206420, 2003.

IPCC: Common Reporting Framework, [online] Available from: https://www.ipccnggip.iges.or.jp/public/gl/guidelin/ch1ri.pdf, 1996.

30 IUPAC: Task Group on Atmospheric Chemical Kinetic Data Evaluation, [online] Available from: http://iupac.pole-ether.fr/, 2019. 